\title{
Health Considerations in the Use of Beverages
}

\author{
Philippa C Ojimelukwe* \\ Department of Food Science and Technology, Nigeria
}

Received: 漹 January 10, 2019; Published: 制January 22, 2019

*Corresponding author: Philippa C Ojimelukwe, Department of Food Science and Technology, Umudike, Nigeria

\begin{abstract}
There are so many beverages to choose from and this may introduce confusion to the consumer. The contribution of a beverage to energy intake, intake of essential nutrients and evidence for positive or negative effects on health benefits can serve as useful guide for the selection of beverages. These are the three factors that may guide an individual in the use of beverages. When investigated based on these three attributes, water emerges the best beverage but there are other healthy beverages. Water helps in regulating body temperature, waste excretion, digestion, circulation and boosting of energy. Drinking water is one of the most ignored healthy habits. Major substances found in other beverages that affect the metabolism of humans include alcohol, caffeine, sugar and taurine. Their metabolism account for the sedative, stimulating and energy boosting effects of beverages. Water should not be completely replaced by other beverages. Consumers should be enlightened on the calorie intake of beverages healthy for the human body. The abuse of the beverages has serious health consequences.
\end{abstract}

Keywords: FAEEs : Fatty Acid Ethyl Esters; ADH: Alcohol Dehydrogenase; ALDH: Aldehyde Dehydrogenase; CYP 2EI: Cytochrome P450 2EI; GABA: Gamma-Aminobutyric Acid

\section{Introduction}

A beverage is any potable liquid, especially one other than water, as tea, coffee, beer, or milk www.thefreedictionary.com/Beverages). Water is abundant, refreshing and adequate to meet body needs for fluids. Potable water is the perfect beverage for quenching thirst and re-hydrating the system. [http://www.nestle-waters.com/ learn-about-water/main-functions-of-water] Food intake, level of activity and the weather affects requirements for water (beer, wine tea, coffee and soft drinks offer pleasure and taste in addition to providing fluids. There are claims that energy drinks provide extra strength (both cognitively and physically). There are three unique uses of water which distinguishes it from beverages. Water is used for breathing, removal of waste, sweating restoration of fluids lost through metabolism. The human body contains up to $60 \%$ water; the brain-75\% water and the blood-83\% water. The amount of water required by the human body is related to food intake, level of activity and weather. For most people, about $80 \%$ of their water intake should come from beverages while the rest comes from food $[1,2]$.

\section{Tea and Coffee}

Tea and coffee are beverages that are very popular worldwide. They are very rich in antioxidants, flavonoids and other bioactive compounds when drunk plain. Plain tea or coffee does not contain calories. Addition of whipped cream, sugar, and flavorings may compromise the health benefits of tea and coffee and increase their calorie content significantly [3].

\section{Milk-based Beverages}

Cow milk and soymilk beverages are suitable for growing children since they contain calcium and vitamin D which are desirable nutrients for bone development. Low fat milk-based beverages are still relatively high in calories and high levels of consumption may increase the risk of prostrate and ovarian cancer. Beverages based on low fat milk contain less calcium. It is best for adults to limit intake of dairy products to a glass or two daily especially when calcium is obtained from other sources.

\section{Sweetened Beverages without Calories}

Diet drinks are sweetened with calorie-free artificial sweeteners. They are better than soft drinks sweetened with sugar but should not be taken daily [4].

\section{Calorie Beverages with Nutrients}

Fruit and vegetable juices, whole milk, mineral enriched water and alcoholic beverages belong to this group. Alcoholic beverages are very popular. They are used extensively in social gatherings, for relaxation, cultural events and the like. Alcohol may have beneficial effects in small quantities but may be hazardous in large amounts for many. Moderate alcohol consumption is said to reduce the risk 
of developing gallstone, type 2 diabetes, dementia, common cold and cardiovascular diseases $[5,6]$. It is believed to raise the level of high-Density Lipoproteins (good cholesterol) and improve libido. Most of these kinds of beverages should not be taken more than one serving per day [7].

\section{Sweetened Caloric Beverages}

Soft drinks, fruit drinks, lemonade, high fructose corn syrup, carbonated beverages, so called energy drinks and flavored coffee/ tea belong to this group. They are the least recommended. They provide a lot of calories without providing other nutrients. Frequent intake of these beverages can lead to weight gain and increase in the risk of type 2 diabetes [8].

\section{Mini Review}

\section{Some constituents of Beverages that affect the Health of Humans}

Alcohol: Alcohol metabolism occurs mainly in the liver. Some alcohol is also metabolized in the pancreas, brain and gastrointestinal tract, causing damage to target cells and tissues [9]. The brain and stomach also metabolizes alcohol. As the major organ responsible for alcohol metabolism, the liver is vulnerable to the negative effects of alcohol metabolism [10]. Heavy drinkers may develop fatty liver while a few of them will eventually suffer from liver cirrhosis. The pancreas is exposed to toxic by-products such as acetaldehyde and Fatty Acid Ethyl Esters (FAEEs) [11]. The major culprit by-product in alcohol metabolism is acetaldehyde (a known carcinogen). Although it eventually gets converted to acetate by aldehyde dehydrogenase, it causes a lot of damage within the brief period when it is available in the human system. Note that a blood alcohol level of 0.3 can be lethal. Alcohol is a sedative drug. It stimulates the secretion of Gamma Amino Butyric Acid (GABA) [12]. Combinations of alcohol and caffeine in some beverages like rum, coke and some so-called energy drinks are common. Drinks containing caffeine and taurine may lead to anxiety, phobias, heart attacks and the like. Energy drinks can mask the effects of alcohol. Energy drinks demonstrate the effects that caffeine and sugar provide but there is no evidence of the effects of other ingredients. GABA is a neurotransmitter that that inhibits other neural processes. When alcohol continues to stimulate the secretion of GABA, important neural and other body systems may become inhibited and may shut down.

Caffeine: Caffeine is a stimulant. It is an antagonist for adenosine, a neurotransmitter. Caffeine inhibits adenosine action thereby increasing levels of arousal and alertness. High levels can produce nervousness, anxiety and tachycardia. Both alcohol and caffeine increase dopamine levels. All drug abuse substances increase dopamine levels. Caffeine can apparently reduce the selective effects of alcohol allowing an individual to drink for longer periods of time However, caffeine does not reduce the impairment of cognitive functions and intoxication associated with the intake of alcohol. It only promotes a false perception that one is less intoxicated than they actually are.

\section{Metabolism of Alcohol and Caffeine}

Alcohol is metabolized in a number of ways. The pathway involves alcohol dehydrogenase $(\mathrm{ADH})$ and aldehyde dehydrogenase (ALDH). Catalase and Cytochrome P450 2EI (CYP 2EI) also metabolize alcohol in smaller quantities [13]. Combined use of caffeine and alcohol increase the rate of alcohol related injury. Caffeine and alcohol are both diuretics and combined use of these substances increases the risk of dehydration. It implies that a depressant is used at the same time as a stimulant. This sends contradictory messages to the nervous system. Adverse effects of drinking heavily include alcoholism, liver damage and various cancers $[14,15]$. Genetic factors such as variations in the quantities of enzymes that metabolize alcohol predispose place some people at greater risks than others. Overall nutrition of an individual also plays a role. Acetaldehyde is highly toxic and carcinogenic [16]. It is metabolized to acetate which is less toxic [16]. Subsequent reactions lead to the conversion of acetate to Carbon dioxide and water. Cytochrome P450 2EI (CYP2 EI) breaks down alcohol to acetaldehyde only when large amounts of alcohol have been consumed. It is possible that CYP 2EI and catalase metabolize alcohol to produce acetaldehyde inside the brain $[17,18]$. Catalase metabolizes only small amounts of alcohol in the body. In addition, small quantities of alcohol interact with fatty acids to form Fatty Acid Ethyl Esters (FAEEs) which cause damage to the liver and pancreas.

\section{Genetics and Alcohol Metabolism}

The amount of alcohol that can be metabolized by an individual every hour depends on genetic factors such as size of the liver and body mass. There are variations in the genes that code for alcohol dehydrogenase and aldehyde dehydrogenase in different individuals. This implies that a fast ADH or a slow ALDH can cause the accumulation of acetaldehyde. Chinese, Japanese and Koreans have a variant of ADH known as ADHIB*2- an enzyme which protects against alcoholism. This enzyme which metabolizes alcohol to acetaldehyde very effectively is rare in Africans and Europeans $[19,20]$.

\section{Caffeine}

Caffeine is absorbed in the small intestines. Its metabolism takes place in the liver and within the next 45 minutes, it is distributed to all tissues in the body. It has a half-life of 3-5 hours in adults but can have a half-life of up to 8 hours in pregnant women. Caffeine breaks down into three major components- paraxanthine $(84 \%$ theobromine $(12 \%)$ and theophylline (4\%). Paraxanthine increases lipolysis leading to elevated glycerol and free fatty acids levels in the blood plasma. Theobromine dilates blood vessels and increases urine volume. Theophylline relaxes smooth muscles in the bronchi $[21,22]$.

\section{Effects of Simultaneous Consumption of Alcohol and Caffeine on the Human Body}

Alcohol is a sedative. It activates the neurotransmitter system. GABA (Gamma-Aminobutyric Acid) is an inhibitory neurotransmitter. When the neurons in the brain release GABA, 
it slows down or inhibits other neural processes. The individual feels more relaxed, reduced anxiety, but at the same time gets sedated. Higher levels of alcohol will increase the inhibition on important neural and other body systems. On the other hand, caffeine is a stimulant. Caffeine is an antagonist for adenosine the (a neurotransmitter). Adenosine is an inhibitory neurotransmitter; similar to GABA, adenosine can inhibit other neural processors. The double negative effect of caffeine (inhibiting an inhibitor of neural processes) leads to increase levels of arousal and alertness-but higher doses can produce nervousness, anxiety and tachycardia [22]. Because the drugs in a sense have opposite effects, one might expect that the simultaneous consumption of both alcohol and caffeine may reduce the effects that are seen when each drug is administered alone, but it is not so. Some effects are reduced while others are increased. The effects also vary with dosage levels (Figures 1-3).

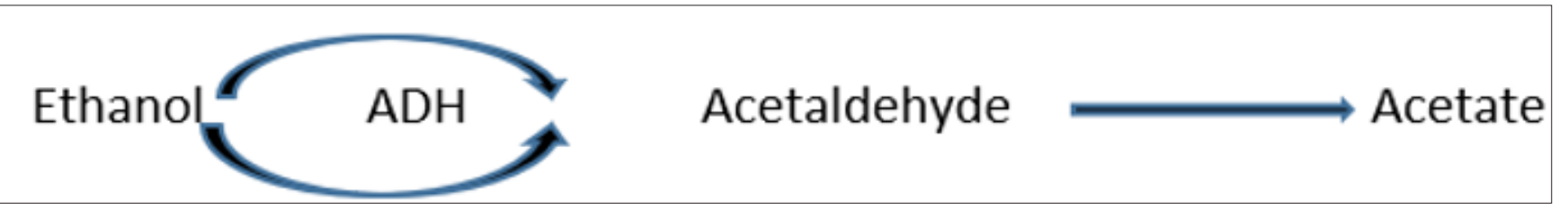

Figure 1: Conversion of ethanol to acetate.

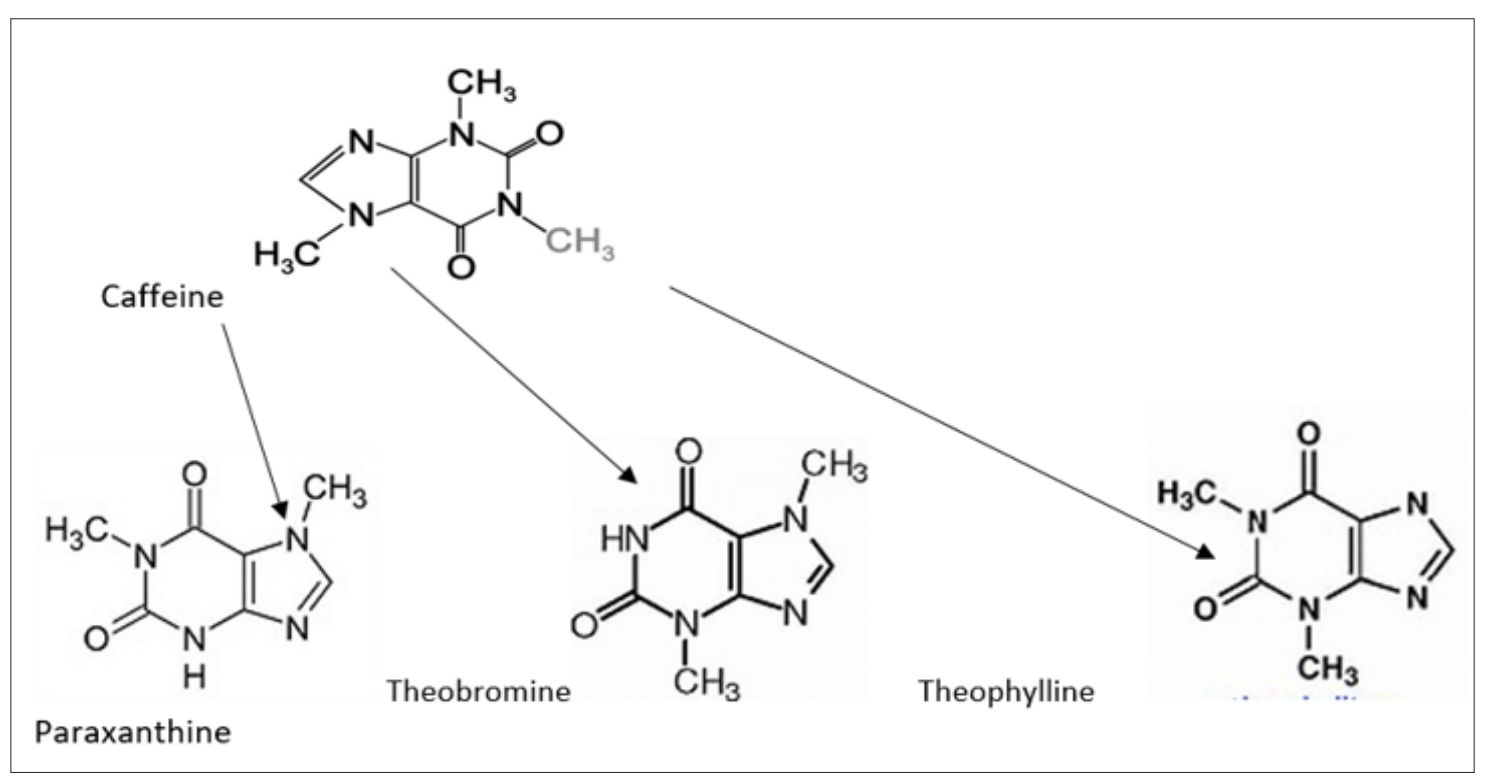

Figure 2: Metabolism of caffeine.

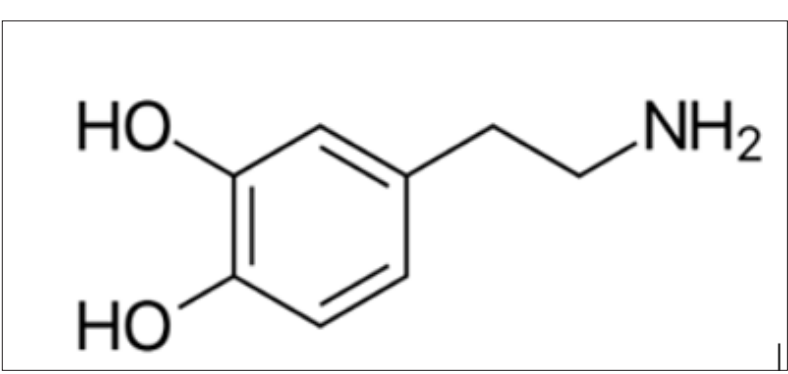

Figure 3: Structure of Dopamine.

\section{Taurine}

Taurine is a free form of amino acid produced in the human body through metabolizing methionine or cysteine. It is now synthetically produced. Taurine is utilized by the body during exercise and in times of stress. It is believed to reduce symptoms of anxiety associated with the intake of caffeine. Energy drinks containing taurine are popular and there are many positive health claims about this conditionally essential amino acid. However, it has been implicated in illnesses like high blood pressure, stroke, seizures and heart diseases. Taurine is involved in the development and function of the brain. It interacts with the neurotransmitters in the regulatory areas of the thalamus. The daily intake of taurine should not be more than 500mg per day [23]. True energy comes from a healthy diet rich in raw foods. A varied diet of wholesome organic foods will supply the taurine requirements of the human body.

\section{Dopamine}

Dopamine is a metabolite associated with the abuse of beverages. Dopamine is a neurotransmitter. It controls reward motivated behavior. Outside the cells, it functions as a local chemical messenger. In the blood vessels, it inhibits the release of norepinephrine and acts as a vasodilator. In the kidneys, it increases 
sodium excretion and urine output. It reduces insulin production and the activity of lymphocytes. Both caffeine and alcohol increase dopamine levels. Dopamine is a neurotransmitter associated with reward. One thing all drugs of abuse have in common is the ability to activate the dopamine system. The ability of alcohol and caffeine to stimulate the dopamine system may be one factor contributing to their use [24].

\section{Recommended Intake of Beverages to maintain Healthy Living}

It is recommended that, at least, half of a man's daily intake of fluids should come from water. One third can come from unsweetened coffee or tea. If tea or coffee is flavored with sugar, cream or whole milk, only little quantities should be taken for proper weight management. A small glass of fruit juice and 1-2 glasses of alcoholic drinks (for men) and 1 glass for women is adequate per day. Diet drinks made with artificial sweeteners should be avoided. Only small quantities of sweetened drinks (e.g. with sugar or high fructose corn syrup should be taken occasionally. Beverages should not constitute more than $10 \%$ of the caloric intake of an individual.

\section{Conclusion}

Beverages are important for the human system. Water should not be completely replaced by beverages. With the proliferation of beverages there is a dire need for nutrition education on proper utilization. The abuse of beverages has serious health consequences.

\section{References}

1. Popkin BM, Armstrong LE, Bray GM, Caballero B, Frei B, et al. (2006) A new proposed guidance system for beverage consumption in the United States. Am J Clin Nutr 83(3): 529-542.

2. Pande R (2018) The new Times. Rwanda's leading Daily.

3. Kuriyama S, Shimazu T, Ohmori K, Kikuchi N, Nnakaya N, et al. (2006) Green tea consumption and mortality due to cardiovascular disease, cancer and all causes in japan: The Ohsaki study. JAMA 296(10): 12551256

4. Gunnars K (2016) Is Diet Soda Bad for You? The Truth About Diet Drinks. Authority Nutrition, 18 September, authoritynutrition.com/why-is-dietsoda-bad-for-you/ Accessed 11 March 2017.

5. Conigrave KM, Hu BF, Camargo CA, Stampfer MJ, Willett WC, et al. (2001) A prospective study of drinking patterns in relation to risk of type 2 diabetes among men. Diabetes 50 (10): 2390-2395.

6. Koppes LL, Dekker JM, Hendriks HF, Bouter LM, Heine RJ (2005) Moderate alcohol consumption lowers the risk of type 2 diabetes: a meta-analysis of prospective observational studies. Diabetes Care 28(3): 719-725.

7. Wiedersehn Sarah (2018) Warning about high sugar in flavoured milk. The Advertiser News Corp Retrieved 9 June
8. Wang YC, Ludwig DS, Sonneville K, Gortmaker SL (2009) Impact of change in sweetened caloric beverage consumption on energy intake among children and adolescents. Archof Pediatr Adolesc Med 163(4): 336-343.

9. Kwo PY, Ramchadani VA, O Conor S Amann D, Carr LG et al. (1998) Gender differences in alcohol metabolism. Relationship to liver volume and effect of adjusting for body mass. Gastroenterology 115(6): 15521557.

10. Ramchandani VA, Bosron WF, Li TK (2001) Research advances in alcohol metabolism Pathologie Biologie 49(9): 676-682.

11. Ammann RW (2001) The natural history of alcoholic chronic pancreatitis. Intern Medi 40(5): 368-375.

12. Mostofsky E, Mukamal KJ, Giovannucci EL, Stampfer MJ, Rimm EB (2016) Key findings on alcohol consumption and a variety of health outcomes from the nurses health study. Am J Public Health 106 (9): 1586-1591.

13. Koop DR (2006) Alcohol metabolism's damaging effects on the cell: A focus on reactive oxygen generation by the enzyme cytochrome P450 2E1. Alcohol Res Health 29(4): 274-280.

14. Kim HJ, Jung S, Eliassen AH, Chen WY, Willett WC, et al. (2017) Alcohol consumption and breast cancer risk in younger women according to family history of breast cancer and folate intake. Am J epidemiol 186(5): 524-31

15. Scoccianti C, Lauby Secretan B, Bello PY, Chajes V, Romieu I (2014) Female breast cancer and alcohol consumption: A review of the literature. Am J Prev Med 46(3): S16-25.

16. Friel PN, Baer JS, Logan BK (1995) Variability of ethanol absorption and breath concentrations during a large-scale alcohol administration study. Alcohol Clinc Exp Res 19(4): 1055-1060.

17. Ting Kai Li, James Beard D, William Orr E, Paul Kwo Y, Vijay Ramchandani A (1998) Gender and ethnic differences in alcohol metabolism. Alcoholism: Clinical and Experimental Research 22(3): 771-772.

18. Neumark YD, Friedlander Y, Durst R, Leitersdorf E, Jaffe D, et al. (2004) Alcohol dehydrogenase polymorphisms influence alcohol-elimination rates in a male Jewish population. Alcohol Clinc Exp Res 28(1): 10-14.

19. Li TK, Beard JD, Orr WE, Kwo PY, Ramchandani VA et al. (2000) Variation in ethanol pharmacokinetics and perceived gender and ethnic differences in alcohol elimination. Alcohol Clin Res 24(4): 415-416.

20. Ehlers CL, Gilder DA, Harris L, Carl L (2001) Association of the ADH2*3 allele with a negative family history of alcoholism in African American young adults. Alcohol Clin Exp res 25: 1773-1777.

21. Heckman MA, Weil J, Gonzalez de Mejia E (2010) Caffeine (1,3,7-trimethylxanthine) in Foods: A comprehensive review on consumption, functionality, safety, and regulatory matters. J Food Sci 75 (3): R77-R87.

22. Ferré S (2016) Mechanisms of the psychostimulant effects of caffeine: Implications for substance use disorders. Psychopharmacology. 233(10): 1963-1979.

23. (2008) Scientists Close In On Taurine's Activity in the Brain. Medical News Today MediLexicon, Intl.

24. Dopamine pathways reward-motivated behavior. 
ISSN: 2574-1241

DOI: 10.26717/BJSTR.2019.13.002395

Philippa C Ojimelukwe. Biomed J Sci \& Tech Res

(c) (i) This work is licensed under Creative

Submission Link: https://biomedres.us/submit-manuscript.php

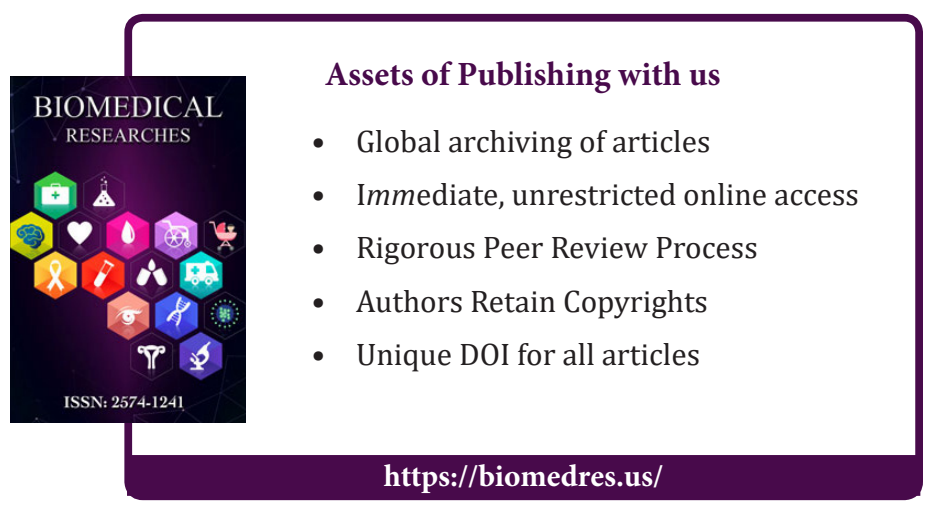

